\title{
PERFIL NUTRICIONAL DE PACIENTES HIV POSITIVO DO MUNICÍPIO DE APUCARANA (PR)
}

\section{Tatiane Bordini Gomes}

Discente da Faculdade de Apucarana (FAP), Apucarana (PR), Brasil.

\section{Natalia Brandão dos Santos Lourival}

Docente da Faculdade de Apucarana (FAP), Apucarana (PR), Brasil

E-mail: tatii_gomes@hotmail.com
RESUMO: Conhecer o perfil nutricional dos pacientes com HIV é o primeiro passo para uma nutrição adequada e um tratamento bem sucedido, garantindo ao paciente uma melhora na qualidade de vida. Sendo assim, o estudo tem por objetivo avaliar o perfil nutricional do paciente portador do Vírus da Imunodeficiência Humana no município de Apucarana(PR). A coleta de dados foi realizada por meio de peso, estatura, índice de massa corporal, circunferência da cintura, circunferência do braço, dobra cutânea tricipital e circunferência muscular do braço. Além disso, foram aplicados questionário de frequência alimentar e questionário sobre histórico da doença. Os resultados apontam uma mudança no perfil nutricional dos pacientes, onde $45,5 \%$ apresentam sobrepeso, seguidos de indivíduos eutróficos. Essa mudança está relacionada ao uso de medicações antirretrovirais, pois estas possuem como efeito colateral a redistribuição de peso corpóreo.

PALAVRAS-CHAVE: AIDS; Antropometria; Avaliação Nutricional.

\section{NUTRITIONAL PROFILE OF POSITIVE HIV PATIENTS IN APUCARANA, BRAZIL}

\begin{abstract}
Knowledge on the nutritional profile of HIV patients is a first step towards an adequate nutrition and successful treatment to guarantee an improvement in life quality. Current analysis assesses the nutritional profile of patients with HIV in Apucarana PR Brazil. Data on weight, height, body mass index, circumference of hips, circumference of arms, tricipital skinfold thickness and muscular circumference of the arm were collected. A questionnaire on food frequency and a questionnaire on the disease were also applied. Results showed a change in the nutritional profile of the patients, revealing that $45.5 \%$ were overweight, followed by eutrophic subjects. Changes are related to anti-retroviral drugs which redistribute body weight as a side effect.
\end{abstract}

KEY WORDS: AIDS; Anthropometry; Nutritional Evaluation.

\section{INTRODUÇÃO}

O Vírus da Imunodeficiência Humana (HIV) ainda é considerado sem cura, porém, nos dias atuais a terapia antirretroviral de alta potência tem se mostrado um tratamento eficiente em relação à sobrevida dos indivíduos soropositivos. A mesma traz mudanças no perfil nutricional do paciente, já que antes o comum era ver mais indivíduos desnutridos, e hoje, devido ao tratamento, a literatura 
vem mostrando que estes pacientes se encontram com sobrepeso ou até mesmo com obesidade (ROCHA, 2007).

O HIV tem como característica um período longo de encubação antes do surgimento dos primeiros sintomas da doença. Esse vírus tem por consequência a Síndrome da Imunodeficiência Adquirida (AIDS), que se caracteriza pelo conjunto de sintomas e sinais da doença até uma enfermidade complexa (BRASIL, 2012).

O principal modo de transmissão do vírus é através do contato sexual, seja ele homossexual ou heterossexual, porém o risco de contrair o vírus é maior com a prática de sexo anal, na ausência de circuncisão, quando há existência de outras DSTs, assim como no período menstrual. A transmissão também pode ocorrer através da exposição ao sangue contaminado, de mãe para filho através da gestação, parto ou aleitamento materno e até mesmo pela recepção de órgão ou tecidos transplantados (SOUZA, 2009).

A célula infectada pelo vírus normalmente é o linfócito $\mathrm{CD} 4$, este direciona muitas outras células da rede imune e com o decorrer da doença o número de linfócito CD4 cai deixando o paciente suscetível a infecções oportunistas. Os macrófagos também são infectados pelo HIV e servem como reservatório para que o vírus possa se disseminar (PAPADAKIS; MCPHEE; RABOW, 2013).

Antes da existência da terapia antirretroviral (TARV) era comum os indivíduos infectados apresentarem caquexia, pois as infecções oportunistas afetam o estado nutricional assim como a febre e o mal-estar podem acarretar em falta de apetite. Enquanto isso as infecções no sistema digestório, comuns nesses pacientes, levam a má absorção, ou até mesmo a um quadro de diarreia crônica, refletindo assim nos sinais de desnutrição acarretados por déficits nutricionais (REIS, 2008).

Apesar de seus efeitos benéficos a terapia antirretroviral também possui efeitos colaterais sendo os mais comuns a resistência à insulina, dislipidemias, hipertensão arterial e consequentemente maior risco de doenças cardiovasculares. Esses efeitos colaterais estão associados a uma redistribuição de gordura corporal nesses pacientes, levando à chamada lipodistrofia (MENDES et al., 2013).

Sendo assim a nutrição desempenha papel importante na vida do indivíduo infectado pelo HIV, seja este usuário da terapia antirretroviral (TARV), ou não. Através de uma alimentação adequada o paciente pode apresentar melhoras no sistema imune, assim como corrigir deficiências nutricionais e/ou alterações metabólicas decorrentes do tratamento (MARRONE, 2008).

Este estudo tem como objetivo avaliar o perfil nutricional do paciente portador do vírus HIV no município de Apucarana (PR).

\section{METODOLOGIA}

A pesquisa foi realizada com o intuito de avaliar o perfil nutricional de pacientes atendidos pelo Núcleo de Aconselhamento Testagem e Tratamento de Apucarana (NATTA), com autorização do secretário de saúde. O NATTA destaca-se no tratamento e atenção do HIV, hepatites virais e outras doenças sexualmente transmissíveis (DSTs). O departamento tem como objetivos reduzir a transmissão destas doenças assim como melhorar a qualidade de vida dos pacientes.

Para participar deste estudo foram incluídos indivíduos de ambos os sexos, portadores do vírus HIV com diagnóstico mínimo de um ano, maiores de 18 anos e que aceitaram participar assinando o Termo de Consentimento Livre e Esclarecido. Foram excluídos indivíduos inconscientes, cadeirantes, acamados e mulheres em gestação, assim como aqueles que não aceitaram responder os questionários propostos ou faltaram nos dias de antropometria.

A coleta de dados foi realizada em uma amostra de 11 indivíduos no período de junho a agosto de 2015, por meio de peso, estatura, índice de massa corporal, circunferência da cintura, circunferência do braço, dobra cutânea tricipital e circunferência muscular do braço, sendo que estes foram registrados em uma tabela antropométrica. Além disso, foram aplicados questionário de frequência alimentar e questionário sobre histórico da doença.

Para isso, o peso foi obtido em uma balança mecânica calibrada da marca Welmy ${ }^{\circledR}$, com capacidade para $150 \mathrm{~kg}$, onde o indivíduo se posicionou em pé, no centro da balança, com roupas leves e sem sapatos 
e adornos. A estatura foi verificada com estadiômetro acoplado à balança com o indivíduo em pé, descalço, com os calcanhares juntos, costas retas, braços estendidos ao lado do corpo, com a cabeça ereta e os olhos fixos à frente, na linha do horizonte (NACIF; VIEBIG, 2011).

Depois de realizado peso e estatura de todos, foi calculado o índice de massa corporal (IMC) dos mesmos. O IMC compreende a relação entre o peso em quilogramas e o quadrado da estatura em metros, cujos resultados foram avaliados de acordo com os pontos de cortes preestabelecidos pela Organização Mundial da Saúde (1997), para os adultos, e de acordo com Lipschitz (1994), para os idosos.

Após o cálculo do IMC foram realizadas as medidas das circunferências. A circunferência da cintura (CC) foi aferida no ponto de menor circunferência, ponto médio da distância entre último arco costal e a crista ilíaca, no sentido horizontal, sem compressão da pele. A circunferência do braço (CB) foi realizada com o antebraço direito do avaliado em posição supinada onde a fita passou por cima do ponto mesumeral (ponto médio entre acrômio e olécrano) (NACIF; VIEBIG, 2011).

Ambas as circunferências foram realizadas com fita antropométrica da marca Incoterm ${ }^{\circledR}$, possuindo material de fibra de vidro inelástica e maleável, com retração automática, trava de fixação e capacidade máxima de 150 centímetros.

Realizou-se também a dobra cutânea tricipital (DCT) no mesmo ponto médio utilizado para realização da CB (entre o acrômio e olécrano), onde foi separada levemente, porém com segurança a prega do braço, desprendendo-a do tecido muscular, e aplicando no indivíduo o adipômetro clínico da marca Cescorf ${ }^{\circledR}$, em ângulo reto, com o braço relaxado e solto ao lado do corpo.

Sendo assim ainda foi avaliado o risco de doenças cardiovasculares (DCV) de acordo com a CC, e utilizados a CB, a DCT e a CMB como indicadores isolados de desnutrição ou obesidade.

Após a antropometria foi aplicado questionário de frequência alimentar adaptado de Ladeira e Silva (2012). Este instrumento avaliou o consumo dos principais grupos alimentares e de alguns alimentos e na obtenção dos resultados foi considerado um hábito alimentar somente aqueles com consumo maior ou igual a 3 vezes por semana.

Os resultados obtidos na frequência alimentar foram utilizados com a finalidade de relacionar o consumo alimentar dos participantes aos resultados obtidos através da antropometria realizada nos mesmos.

Por fim, foi aplicado um questionário a fim de obter informações sobre o histórico da doença, como o tempo de diagnóstico e o uso de medicamentos antirretrovirais, pois estes fatores podem causar alterações no perfil nutricional do indivíduo, que, segundo Diehl et al. (2008), acontece com $65 \%$ dos pacientes portadores do vírus HIV, podendo assim alterar os resultados obtidos.

Os dados foram tabulados em planilhas no programa Microsoft Office Excel, versão 2007.

A pesquisa foi aprovada pelo Comitê de Ética envolvendo Seres Humanos, de acordo com o parecer $\mathrm{n}^{\circ}$ 1.314.639, além de respeitar a Resolução no 466, de 12 de dezembro de 2012.

\section{RESULTADO E DISCUSSÃO}

A pesquisa foi realizada com uma amostra de 11 indivíduos, sendo 03 do sexo masculino e 08 do sexo feminino, o que corresponde a 27,3 e $72,7 \%$, respectivamente. A faixa etária dos mesmos variou de 29 a 65 anos. O pequeno número de participantes se dá pelo fato da baixa quantidade de indivíduos portadores de HIV que estão frequentando o NATTA atualmente, sendo assim 11 participantes representam $73,3 \%$ do total de indivíduos. Os outros 26,7\% (04 indivíduos) não aceitaram participar da pesquisa.

Resultados diferentes foram encontrados em estudo realizado por Rodrigues, Miranda e Guterres (2013), onde a maior parte da amostra infectada foi do sexo masculino (60\%), o mesmo acontece nos resultados encontrados por Leite e Sampaio (2011), que também destacavam mais casos de homens (63\%) do que de mulheres infectadas.

Porém, de acordo com Garcia e Souza (2010), desde os anos 90 o número de casos de HIV na população feminina vem aumentando consideravelmente, 
principalmente no Brasil, ocasionando uma mudança no perfil da epidemia, o mesmo ocorre com o aumento do número de casos entre pessoas com 30 anos ou mais.

Em relação ao estilo de vida dos indivíduos, $100 \%$ são heterossexuais e fazem uso de medicamentos antirretrovirais, sendo que $63,6 \%$ relatam ter contraído o vírus através de relação sexual desprotegida e o restante dos indivíduos não possuem conhecimento de como foram infectados. Nota-se também que mais da metade dos indivíduos $(72,8 \%)$ contraiu o HIV há mais de 06 anos, porém quando analisado o tempo de uso de medicação, $45,5 \%$ destes só fazem uso de terapia antiretroviral há menos de 05 anos. Neste tempo apenas uma paciente precisou interromper o tratamento sendo que esta o retomou posteriormente (Tabela 1).

Tabela 1. Caracterização do estilo de vida, histórico da doença e uso de medicações

\begin{tabular}{|c|c|c|}
\hline & $\mathrm{N}^{\mathrm{O}}$ & $\%$ \\
\hline \multicolumn{3}{|l|}{ Orientação sexual } \\
\hline Heterossexual & 11 & $100 \%$ \\
\hline Homossexual & 0 & $0 \%$ \\
\hline Bissexual & 0 & $0 \%$ \\
\hline \multicolumn{3}{|l|}{ Forma de contração do vírus HIV } \\
\hline Relação sexual desprotegida & 7 & $63,6 \%$ \\
\hline Nascimento/amamentação & 0 & $0 \%$ \\
\hline Sangue contaminado (transfusão/seringa) & 0 & $0 \%$ \\
\hline Não Sabe & 4 & $36,4 \%$ \\
\hline Outro & 0 & $0 \%$ \\
\hline \multicolumn{3}{|l|}{ Tempo de diagnóstico da doença } \\
\hline Menos de 1 ano & 0 & $0 \%$ \\
\hline 1 a 5 anos & 3 & $27,3 \%$ \\
\hline 6 a 10 anos & 3 & $27,3 \%$ \\
\hline 11 a 15 anos & 3 & $27,3 \%$ \\
\hline Mais de 15 anos & 2 & $18,2 \%$ \\
\hline \multicolumn{3}{|l|}{ Uso de antirretrovirais } \\
\hline Sim & 11 & $100 \%$ \\
\hline Não & 0 & $0 \%$ \\
\hline \multicolumn{3}{|l|}{ Tempo de uso de antirretrovirais } \\
\hline Menos de 1 ano & 0 & $0 \%$ \\
\hline 1 a 5 anos & 5 & $45,5 \%$ \\
\hline 6 a 10 anos & 3 & $27,3 \%$ \\
\hline 11 a 15 anos & 2 & $18,2 \%$ \\
\hline Mais de 15 anos & 1 & $9,1 \%$ \\
\hline \multicolumn{3}{|l|}{ Interrupção no tratamento } \\
\hline 1 a 6 meses & 1 & $9,1 \%$ \\
\hline 6 a 12 meses & 0 & $0 \%$ \\
\hline Mais de 1 ano & 0 & $0 \%$ \\
\hline Não houve interrupção & 10 & $90,9 \%$ \\
\hline
\end{tabular}

Fonte: Natta (2015).
Pesquisa semelhante realizada em 2009, por Neto et al., também relata que a maior parte dos indivíduos infectados são heterossexuais (84\%), e que a forma de contaminação mais descrita foi através de relação sexual desprotegida $(82,5 \%)$. Isso também ocorreu em pesquisa realizada por Silva et al. (2009), que demonstra o aumento de casos de infecção através de relação sexual sem proteção, condizendo assim com os resultados encontrados no presente trabalho.

O principal motivo do grande aumento de indivíduos heterossexuais com HIV se dá pelo fato de que na fase inicial da descoberta da epidemia apenas indivíduos homossexuais eram considerados um grupo de risco para infecção, sendo assim os indivíduos heterossexuais acreditaram ser imunes à doença $\mathrm{e}$ deixaram de tomar as devidas precauções para evitar o contágio (NODIN; CARBALLO-DIEGUEZ; LEAL, 2015).

$O$ fato de muitos dos pacientes fazerem uso de terapia antirretroviral há menos de 05 anos e terem contraído o vírus há um tempo maior, provavelmente acontece pois, normalmente, a medicação só é indicada para pacientes com contagem de CD4 abaixo de $500 \mathrm{cel} /$ $\mathrm{mm}^{3}$, acabando por adiar o início do tratamento. Porém, de acordo com Coelho (2015), nos primeiros meses de 2015 o Ministério da Saúde registrou um acréscimo de mais de $40 \%$ no número de pessoas em tratamento com CD4 superior a $500 \mathrm{cel} / \mathrm{mm}^{3}$, o que tende a facilitar o acesso dos pacientes aos medicamentos.

Ao se avaliar apetite, 54,5\% dos indivíduos relataram não possuir alteração nos últimos meses, enquanto o restante apresentou algum tipo de alteração onde 27,3 e 18,2\% relataram redução de apetite e aumento de apetite, respectivamente.

Logo, a alteração de apetite foi relatada por quase metade da amostra. Isso pode ocorrer devido à própria infecção pelo HIV, assim como pelo uso de medicamentos antirretrovirais (RIBEIRO, 2010). A redução de apetite acaba sendo um sintoma mais frequente, porém muitos dos indivíduos ainda apresentam sintoma oposto (SANTOS, 2012).

Resultado semelhante foi visto por Cunha (2011) onde, em uma amostra de 100\% dos indivíduos em uso de TARV, aproximadamente metade relatou alteração de apetite. Neste estudo a quantidade de indivíduos 
que relatou redução de apetite (36\%) foi maior do que a quantidade que relatou aumento (9\%), assim como ocorreu na atual pesquisa.

Com relação à composição corporal, (Gráfico 1) a maior parte dos indivíduos encontra-se com IMC de sobrepeso (45,5\%), seguido de indivíduos eutróficos (36,4\%). Nenhum dos indivíduos apresentou desnutrição. Provavelmente não houve casos de baixo peso, pois vale ressaltar que todos os participantes fazem uso de medicamentos antirretrovirais, o que leva a modificações no perfil nutricional, diminuindo assim as chances de ocorrer uma desnutrição e aumentando as chances de ganho de peso.

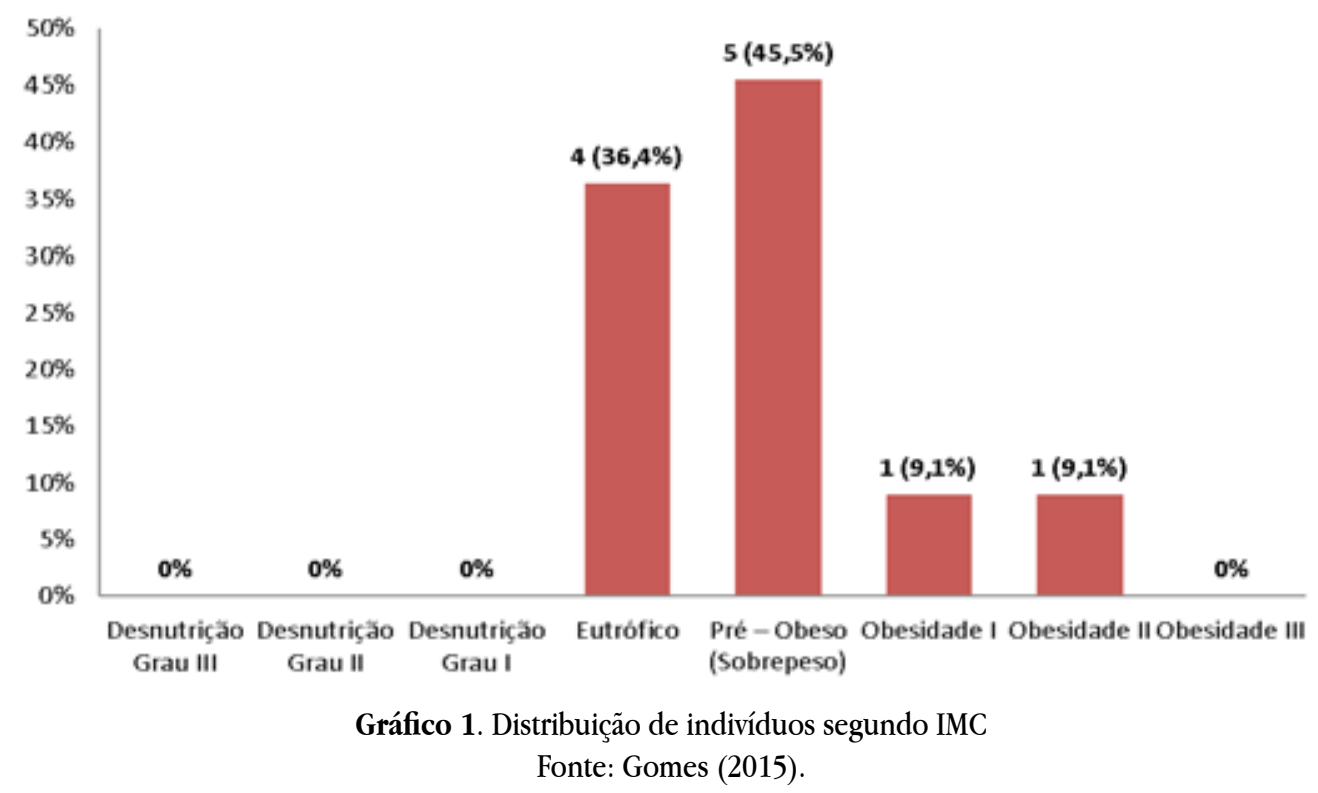

Capello et al. (2010) realizaram um estudo sobre o estado nutricional de pacientes com HIV e o mesmo demonstrou que os participantes apresentavam, em sua maioria, IMC de sobrepeso, o que coincid com os resultados obtidos nesta pesquisa. Já o trabalho realizado no mesmo ano por Macedo e Guimarães apresenta resultados diferentes, onde $87 \%$ dos pacientes foram classificados como eutróficos, mostrando um melhor perfil nutricional.

Sampaio (2012) também apresentou maior percentual de indivíduos eutróficos em seus resultados $(54,8 \%)$, porém a taxa de indivíduos com excesso de peso não deixa de ser significativa, totalizando $39,8 \%$ da amostra.

Marrone (2008) obteve dados muito parecidos com os da atual pesquisa, onde $50 \%$ dos indivíduos apresentaram sobrepeso, seguidos de 33,3\% de casos de eutrofia e finalizando com 16,4\% de casos de obesidade. Os resultados muito semelhantes ao presente trabalho podem ser justificados pelo número de participantes,

considerando que Marrone também utilizou uma quantidade baixa, de apenas 06 indivíduos.

Foi analisado ainda o risco de DCV através da CC, e constatou-se que $63,6 \%$ dos indivíduos possuem risco muito aumentado, enquanto o restante $(36,4 \%)$ está com CC adequada. Possivelmente essa grande quantidade de indivíduos com CC elevada acontece, pois, como já citado, pacientes em uso de TARV tendem a sofrer uma alteração em sua composição corporal, sendo uma dessas alterações o acúmulo de gordura na região abdominal.

Silva, Mori e Guimarães (2012) também analisaram a medida de CC em pacientes com HIV e encontraram um percentual de $18,6 \%$ de pacientes com risco muito elevado para DCV. Braga e Silva (2010) não se distanciaram desse resultado, encontrando $22,8 \%$ de sua amostra com CC muito elevada. Mesmo ambos apresentando percentual relativamente inferior ao encontrado no presente trabalho, esses valores não deixam de ser importantes.

Quando comparados os valores de IMC aos resultados obtidos através da circunferência da cintura, 
nota-se que existe uma relação entre o excesso de peso e o aumento de risco para DCV. Nota-se também que, quando se analisa o sexo, as mulheres apresentam maior IMC e maior risco segundo CC do que os homens (Gráfico 2).

Turushima, Ferreira e Bennemann (2015) realizaram uma pesquisa comparando a relação entre IMC e CC, onde a mesma também foi feita com adultos de ambos os sexos, porém sem a presença do vírus HIV. Mesmo assim os resultados obtidos são semelhantes aos obtidos no atual trabalho, mostrando correlação positiva e significativa do IMC com a CC.
Ladeira e Silva (2012) também verificaram que as mulheres apresentavam maior risco de doenças metabólicas do que os homens, assim como Diehl et al. (2008), que estudaram pacientes atendidos no Ambulatório de Infectologia/AIDS da Universidade Estadual de Londrina. Enquanto isso, Jaime (2004) observa em sua amostra que os valores de IMC também foram elevados nos indivíduos do sexo feminino. Esses dados são similares aos desta pesquisa, porém deve ser levado em conta que no presente trabalho o número da amostra é pequeno, o que pode causar distorção de resultados.

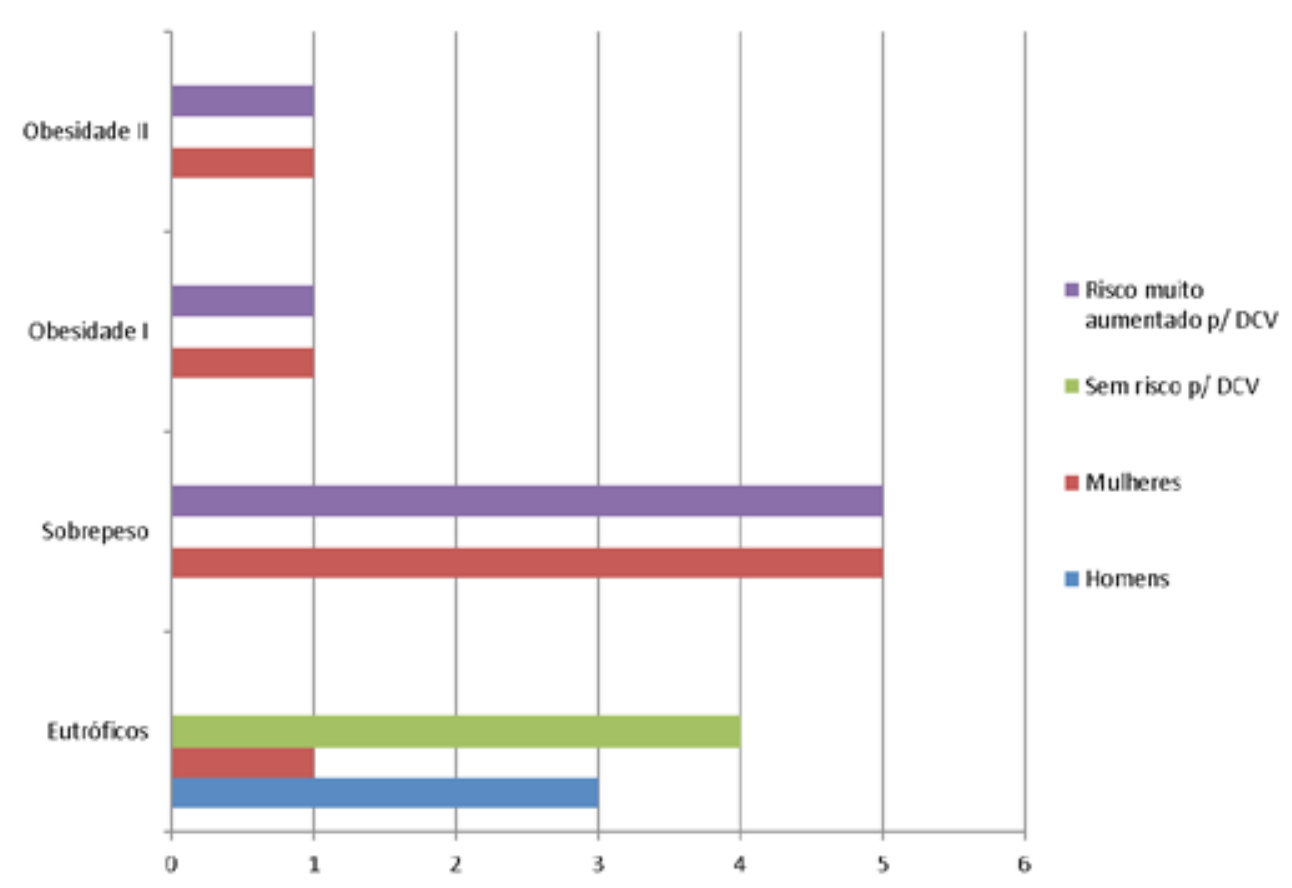

Gráfico 2. Relação entre IMC, risco de doenças cardiovasculares e sexo dos indivíduos Fonte: Gomes (2015).

Foi avaliado também o estado nutricional dos indivíduos de maneira isolada, através da CB e da DCT, além da reserva de degradação de massa magra dos mesmos através da $\mathrm{CMB}$, sendo que por estes parâmetros, a maior parte dos indivíduos foi classificada como eutróficos, havendo poucos casos de desnutrição ou deficiência de massa magra (Gráfico 3). Não foram encontras relações específicas com a classificação destes parâmetros e a classificação segundo IMC.

Braga e Silva, ao avaliarem a CB de indivíduos com HIV, encontraram a maior parte classificados como eutrofia (41\%), o mesmo aconteceu na adequação de CMB onde $61,4 \%$ dos pacientes também se encontravam eutróficos, esses resultados condizem aos encontrados no atual trabalho, apenas se diferem quando comparados à DCT, pois nessa medida Braga e Silva classificaram grande parte de indivíduos como desnutrido grave $(55,7 \%)$, o que não acontece na presente pesquisa.

Ferreira (2008) avaliou o estado nutricional de pessoas vivendo com HIV no Estado de Mato Grosso do 


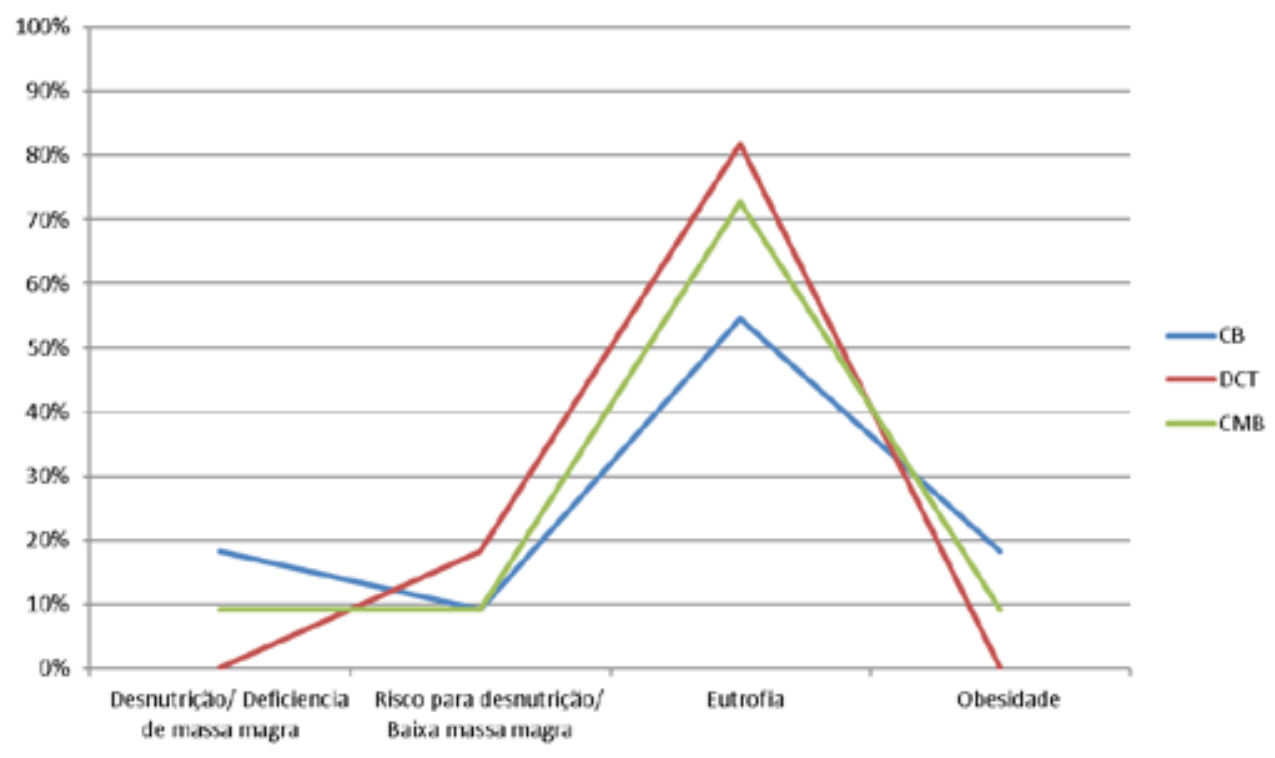

Gráfico 3. Estado nutricional dos indivíduos segundo CB, DCT e CMB Fonte: Gomes (2015).

Sul e, utilizando a medida de CB, 52,3\% dos indivíduos avaliados apresentaram eutrofia, dado esse muito semelhante ao encontrado atualmente.

Resultados diferentes foram encontrados por Silva, Mori e Guimarães (2012), onde através da CBM 64,3\% dos pacientes estavam com déficit de adequação; em relação à DCT 41,6\% dos indivíduos apresentaram desnutrição e o mesmo aconteceu ao avaliarem a CB, onde foi verificado que $65,5 \%$ da amostra também foram classificados com este diagnóstico. Isso demonstra que apesar da mudança que a TARV traz ao perfil nutricional do indivíduo infectado, os mesmos ainda estão sujeitos a complicações nutricionais como a desnutrição e a perda de massa magra.

Através do questionário de frequência alimentar foi possível verificar quais alimentos e grupos alimentares eram hábitos dos pacientes. E o que verificou-se foi que em relação à alimentação, os mesmos apresentam um bom hábito alimentar, com exceção de poucos casos (Gráfico 4). Também foi observado que $100 \%$ dos pacientes não utilizam o azeite e, em contrapartida, todos possuem hábito alimentar de consumir outros tipos de óleos, isso possivelmente ocorre pois, apesar de não ser realizado um questionário específico, foi observado que a maior parte dos indivíduos possui baixa renda e dependem muito de cesta básica para alimentação. Sendo assim, existe certa dificuldade na compra do azeite. Por outro lado, o consumo de óleo habitual pode ser justificado, pois o mesmo é utilizado no preparo das refeições como arroz e carnes.

Verifica-se que o grupo de indivíduos em questão possuiu consumo regular de leite e derivados, carne, peixes e ovos, óleos, pão, arroz e massas, hortaliças e legumes, frutas e café, pois através do questionário foi visto que estes são consumidos 03 ou mais vezes por semana.

Ladeira e Silva (2012), em pesquisa sobre estado nutricional e perfil alimentar de indivíduos com HIV, também observaram que leite e derivados, carnes, óleos vegetais, pão, arroz, hortaliças, legumes, frutas e café faziam parte do hábito alimentar dos indivíduos, pois estes eram consumidos 04 ou mais vezes por semana.

No entanto, de acordo com os resultados de Dutra et al. (2012), foi encontrado o consumo insuficiente de legumes e verduras e consumo elevado de bebidas alcoólicas, doces e guloseimas, dados esses que não foram observados neste trabalho.

\section{Rodrigues, Miranda e Guterres}

constataram que os indivíduos analisados também não faziam uso de azeite e possuíam renda baixa, coincidindo com os resultados aqui encontrados.

É válido ressaltar que o uso de questionário de frequência alimentar pode ter subestimado ou superestimado a quantidade de determinados alimentos consumidos. Além disso, de acordo com os resultados 
de IMC e o questionário aplicado, observou-se que não houve associação entre hábito alimentar e estado nutricional, considerando que os participantes possuem hábito de consumir arroz, leite, frutas e verduras diariamente e não possuem hábito de consumir doces, refrigerantes, bebidas alcoólicas e comidas fora de casa. Em contrapartida, quando comparadas as demais medidas realizadas (CB, PCT, DCT), os hábitos alimentares refletem no perfil nutricional dos indivíduos que é, em sua maioria, de eutróficos.

\section{CONCLUSÃO}

Mediante ao que foi exposto, pode-se concluir que a mudança no perfil nutricional dos indivíduos soropositivos foi elevada, e que existe uma relação entre o sexo feminino e os indicadores antropométricos IMC e CC.

Constatou-se também que, apesar do excesso de peso, os participantes possuíam bons hábitos alimentares, portanto a terapia antirretroviral tende a ser a principal

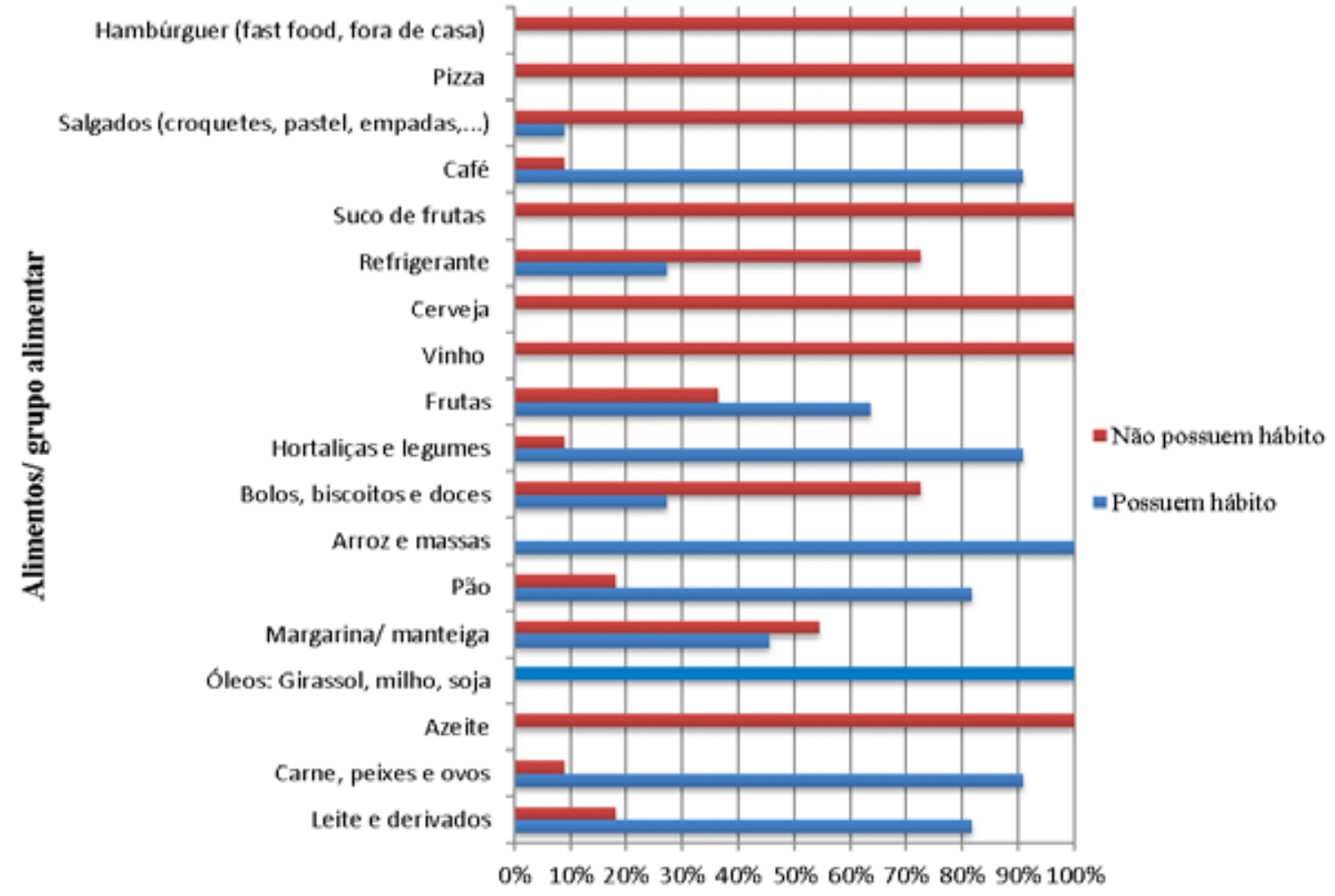

Gráfico 4. Distribuição de indivíduos segundo hábitos alimentares Fonte: Gomes (2015).

responsável pelas alterações no perfil nutricional.

Vale ressaltar que mesmo com investimentos feitos pela área da saúde em iniciativas de prevenção contra a doença, muita gente ainda não toma os devidos cuidados ou não possui instrução suficiente de como se prevenir, já que a relação sexual desprotegida continua sendo a principal forma de transmissão.

Sendo assim, os profissionais da área de nutrição devem estar integrados às equipes multiprofissionais para que possam avaliar o paciente e diagnosticar o perfil nutricional do mesmo, e dessa forma fazer uma intervenção e um acompanhamento específico e individualizado a fim de melhorar o prognóstico da doença. 


\section{REFERÊNCIAS}

BRAGA, L. A.; SILVA, C. A. B. Avaliação nutricional e metabólica de pacientes com HIV em uso de terapia antirretroviral no nordeste do Brasil. Rev Bras em Promoção da Saúde. v. 23, n. 4, p. 368-373, out./dez., 2010.

BRASIL. Ministério da Saúde. Secretaria de Vigilância em Saúde. Departamento de DST, Aids e Hepatites Virais. Recomendações para a prática de atividades físicas para pessoas vivendo com HIV e AIDS. Brasília: Ministério da Saúde, 2012.

CAPELLO, A. C.; SILVA, T. C. V.; DEMINICE, R.; ROSA, F. T. Avaliação do estado nutricional e perfil lipídico de portadores do vírus da imunodeficiência humana (HIV). In: SIMPÓSIO NACIONAL DE INICIAÇÃO CIENTÍFICA, 4., 2014, Londrina. Anais... Londrina: Unifil, 2014.

COELHO, N. Novo protocolo aumenta acesso ao tratamento com antirretrovirais. Disponível em: $<$ http://portalsaude.saude.gov.br/index.php/cidadao/ principal/agencia-saude/18211-novo-protocolo-aumentaacesso-ao-tratamento-com-antirretrovirais $>$. Acesso em: 20 ago. 2015.

CUNHA, A. R. H. Implicações do tratamento com antiretrovirais de alta potência no estado nutricional e qualidade de vida. 2011. 78f. Dissertação (Mestrado em Políticas Públicas) - Escola Superior de Ciências da Santa Casa de Misericórdia de Vitória, Vitória, 2011.

DIEHL, L. A.; DIAS, J. R.; PAES, A. C. S.; THOMAZINI, M. C.; GARCIA, L. R.; CINAGAWA, E.; WEICHMANN, S. L.; CARRILHO, A. J. F. Prevalência da lipodistrofia associada ao HIV em pacientes ambulatoriais brasileiros: relação com síndrome metabólica e fatores de risco cardiovascular. Arq Bras Endocrinol Metab, São Paulo, v. 52, n. 4, p. 658-667, jun. 2008

DUTRA, C. D. T.; MOURA, A. K. M.; SARAIVA, D. A.; DIAS, R. M.; OLIVEIRA, S. H. S. D.; FRAZÃO, A. G. F.; LIBONATI, R. M. F. Fatores de risco para doenças cardiovasculares em pacientes com síndrome lipodistrófica do HIV. Rev Eletr Gestão \& Saúde, v. 3, n. 3, p. 836-851, 2012.
FERREIRA, R. S. Estado nutricional de pacientes vivendo com HIV/AIDS atendidos no hospital universitário da Universidade Federal de Mato Grosso do Sul. 2008. 118f. Dissertação (Mestrado em Saúde e Desenvolvimento) - Universidade Federal de Mato Grosso do Sul, Campo Grande, 2008.

JAIME, P. C. Prevalência de sobrepeso e obesidade abdominal em indivíduos portadores de HIV/AIDS, em uso de terapia antiretroviral de alta potência. Rev. Bras. Epidemiol. v. 7, n. 1, 2004.

LADEIRA, P. O. C.; SILVA, D. C. G. Estado Nutricional e Perfil Alimentar de Pacientes Assistidos pelo Programa de DST/AIDS e Hepatites Virais de um Centro de Saúde de Itaperuna- RJ. DST - J bras Doenças Sex Transm, v. 24, n. 1, p. 28-31, 2012.

LEITE, L. H. M.; SAMPAIO, A. B. M. M. Risco cardiovascular: marcadores antropométricos, clínicos e dietéticos em indivíduos infectados pelo vírus HIV. Rev. Nutrição, Campinas, v. 24, n. 1, p. 79-88, jan./fev. 2011.

MACEDO, V. S.; GUIMARÃES, E. M. A. Perfil antropométrico de indivíduos atendidos pelo programa DST- AIDS de um município do vale do Aço, MG. NUTRIR GERAIS, Ipatinga, v. 4, n. 6, p. 593-603, fev./jul. 2010.

MARRONE, L.; SANTA, A. G.; MARIOTO, C. S. P.; MARTA, C. F. P.; MOTERANI, G. M. B.; NEGRI, E. P.; MIRA, E. C. S. Análise do prognóstico de pacientes infectados com HIV de Londrina/PR e região de acordo com perfil nutricional. 3., 2009, Londrina. Anais eletrônicos... Londrina: UNIFIL, 2009. Disponível em: <http://www.unifil.br/ III_Encontro_Extensao/Sumario.asp $>$. Acesso em: nov. 2015.

PAPADAKIS, M. A.; MCPHEE, S.; RABOW, M. W. Current Medicina: diagnóstico e tratamento. $51^{\mathrm{a}}$ ed. Porto Alegre: AMGH, 2013.

MENDES, E. L.; ANDAKI, A. C. R.; AMORIM, P. R. S.; NATALI, A. J.; BRITO, C. J.; PAULA, S. O. Treinamento físico para indivíduos HIV positivo submetidos à HAART: efeitos sobre parâmetros antropométricos e funcionais. Rev Bras Med Esporte, São Paulo, v. 19, n. 1, p. 16-21, fev. 2013. 
NACIF, M.; VIEBIG, R. F. Avaliação antropométrica no ciclo da vida: uma visão prática. $2^{\mathrm{a}}$ ed. São Paulo: Metha, 2011.

RODRIGUES NETO, J. F.; LIMA, L. S; ROCHA, L. F.; LIMA, J. S.; SANTANA, K. R.; SILVEIRA, M. F. Perfil de adultos infectados pelo vírus da imunodeficiência humana (HIV) em ambulatório de referência em doenças sexualmente transmissíveis no norte de Minas Gerais. Rev. méd. Minas Gerais, v. 20, n. 1, jan./mar. 2010.

NODIN, N.; CARBALlO-DIEGUEZ, A.; LEAL, I. P. Comportamentos sexuais de risco e preventivos masculinos: resultados de uma amostra recolhida através da internet em Portugal. Saúde soc., São Paulo, v. 24, n. 2, p. 607-619, jun. 2015.

OLIVEIRA, O. M. V.; MEDEIROS, R. S.; NASCIMENTO, M. A. B.; BONI, M. S. Perfil nutricional e fatores de risco para obesidade central de pessoas que vivem com HIV/AIDS. Com. Ciências Saúde, Brasília, v. 19, n. 4, p. 305-314, out. 2008.

REIS, L. C. Perfil nutricional de crianças e adolescentes portadores de HIV em acompanhamento ambulatorial. 2008. 109f. Dissertação (Pós-Graduação em Saúde Pública) - Universidade de São Paulo, São Paulo, 2008.

RIBEIRO, C. S. A. Prevalência de alterações nutricionais e fatores de risco para desnutrição em pacientes hospitalizados por HIV/AIDS. 2010. 82f. Dissertação (Mestrado em Alimentos, Nutrição e Saúde) Universidade Federal da Bahia, Salvador, 2010.

ROCHA, P. B. Perfil alimentar e nutricional dos pacientes HIV positivo atendidos em um serviço público de saúde de Porto Alegre/RS. 2007. 47f. Monografia (Nutrição) - Universidade Federal do Rio Grande do Sul, Faculdade de Medicina, UFRGS, 2007.

RODRIGUES, E. C.; MIRANDA, R. N.; GUTERRES, A. S. Avaliação do perfil nutricional e alimentar de portadores do HIV. Rev Para Med., v. 27, n. 4, out./dez. 2013.

SAMPAIO, E. J. Ângulo de fase em pacientes com HIV/AIDS: comparação com métodos de avaliação do estado nutricional. 2012. 85f. Dissertação (Mestrado em Alimentos, Nutrição e Saúde) - Universidade Federal da Bahia, Salvador, 2012.

SANTOS, L. Indicadores antropométricos de gordura corporal no auxílio ao diagnóstico da Síndrome Lipodistrófica do HIV (SLHIV). Pelotas: UFPel; ESEF, 2012.

SILVA, L. C. F.; SANTOS, E. M.; SILVA NETO, A. L.; MIRANDA, A. E.; TALHARI, S.; TOLEDO, L. M. Padrão da infecção pelo HIV/AIDS em Manaus, Estado do Amazonas, no período de 1986 a 2000. Rev. Soc. Bras. Med. Trop., Uberaba, v. 42, n. 5, p. 543-550, out. 2009.

SILVA, V.; MORI, R. M.; GUIMARÃES, S. Alterações nutricionais em pacientes com lipodistrofia associada ao HIV/AIDS de uma unidade de referência do município de Belém - Pará. J bras Doenças Sex Transm. v. 24, n. 4, p. 233-238, 2012.

SOUZA, R. G. Perfil Nutricional de portadores do HIV e ou AIDS e sua correlação com a TARV, na cidade de Belém, Pará, Brasil. 2009. 109f. Dissertação (Mestrado em Biologia de Agentes Infecciosos e Parasitários) Universidade Federal do Pará, Instituto de Ciências Biológicas, Belém, 2009.

TURUCHIMA, M. T.; FERREIRA, T. N.; BENNEMANN, R. M. Associação entre indicadores antropométricos (IMC e CC) em relação ao risco para doenças cardiovasculares. SaudPesq, v. 8, n. Edição Especial, p. 55-63, 2015.

Recebido em: 16 novembro 2015 Revisado em: 11 fevereiro 2016 Aceito em: 29 fevereiro 2016 